\title{
Mechanically Robust Re-crosslinkable Polymeric Hydrogels for Water Management of Void Space Conduits Containing Reservoirs
}

\author{
Lizhu Wang*, Yifu Long, Haifeng Ding, Jiaming Geng, Baojun Bai*
}

\begin{abstract}
A unique preformed particle gel with re-crosslinkable ability to re-form bulk gel for chemical enhanced oil recovery of void space conduits containing reservoir is described herein. The formation of the bulk gel was achieved by reversible ionic crosslinks of the preformed particle gels as induced by brine under reservoir conditions. The re-crosslinked hydrogel with ionic features showed rubber-like mechanical behaviors, a critical parameter of the particle gels to increase plugging efficiency. The re-crosslinking ability of the preformed particle gels under different salinity could be controlled by tuning the ionic interactions within the hydrogel. In addition, the long-term thermal stability of the re-crosslinked bulk gel has been demonstrated. Furthermore, the re-crosslinked bulk gels could significantly reduce permeability of large fractures as demonstrated by core flooding experiments compared with traditional preformed particle gels. The re-crosslinkable bulk gel with robust mechanical properties could offer the candidate to block void space conduits containing reservoirs for enhanced oil recovery.
\end{abstract}

Key words: particle gel, re-crosslinking, enhanced oil recovery 


\section{Introduction}

Gel treatment, an economically and technically viable enhanced oil recovery (EOR) technique, was deployed to increase conformance and reduce the channeling from fractures, fracture-like features and voids in water and gas flooding.[1] Treatment of fractures by gels could divert water or gas penetration through high permeability zones/fractures while maintaining production zone intact. In-situ crosslinked gels formed under reservoir conditions and preformed particle gels (PPGs) prepared at a surface facility prior to injection were widely employed for control conformance to increase oil recovery and reduce water production.[2] During in-situ gel treatment, the polymers and crosslinkers were simultaneously injected to target formation where the gel was formed under reservoir environments to seal the formation.[3] However, there are some potentially intrinsic drawbacks of gelling kinetics and gelation quality due to shear degradation, chromatographic fractionation and formation water dilution.[4] Moreover, in-situ generated gels with weak metallic crosslinks had poor mechanical integrity, vulnerable to break through by water or gas under high pressures. Single component PPGs, including dispersed particle gel, in the sizes of nano- to milli-meters provided an alternative option to circumvent water dilution and mechanical vulnerability.[5-7] Furthermore, the ease in injection using produced water and elastic, deformable characteristics rendered PPGs penetrate to the formation of far wellbore compared to conventional in-situ gels. Field applications in China have demonstrated that millimeter-sized PPGs can significantly reduce the permeability of fractures containing oil reservoirs.[8] In particular, successful PPG treatment by Occidental Oil Company has been applied to control $\mathrm{CO}_{2}$ breakthrough in the course of $\mathrm{CO}_{2}$ flooding.[9-11] However, traditional PPGs were applied to small fractures or low permeability containing reservoirs due to their inability to form bulk gel after placed in void space conduits (VSC) 
reservoirs. They do not have the ability to penetrate the porous rock matrix, particularly the low permeability fractures due to their relatively greater size.

High permeabilities from fracture-like freaks in carbonate fractured oil reservoirs pose great challenges to the oil production in petroleum industry while carbonate reservoirs constitute substantially to US oil reservoirs.[12] However, the low oil recovery from VSC carbonate reservoirs in the US and the world necessitates the development of re-crosslinkable PPGs to increase plugging efficiency for chemical EOR. Rapid breakthrough of water and $\mathrm{CO}_{2}$ during the flooding has been recognized as a severe problem due to the formation of the high-permeability streaks.[13] The streaks as the main form of reservoir heterogeneities consist of open fractures and fracture-like features of conduits, worm holes and caves.[14] Open hole fractures have been demonstrated to significantly reduce the oil recovery because the displacing fluid would bypass oil-rich unswept areas/zones.[15-16] For example, the rapid breakthrough in the Anton Irish Clearfork flood has resulted in the shut-in wells in which large void space flow conduits were generated from flooding induced and enhanced fractures. Moreover, the excess water and $\mathrm{CO}_{2}$ production had the wells uncompetitive compared to its original high oil recovery. Furthermore, in-situ crosslinked gel treatment proved to be fruitless in the Anton Irish field due to its low mechanical strength.[17] In addition, milimeter-sized PPGs have been successfully applied to the reservoirs having fractures and fracture-like features.[18] However, as demonstrated in previous research, PPGs were ineffective in high permeability VSC containing reservoirs.[19] It is therefore necessary to develop new preformed particle gels with re-crosslinkable properties to efficiently plug high permeability zones.

Recent progress in synthetic hydrogels for industry applications requires mechanical robustness and self-healing properties, re-constructing the bulk materials from the relatively 
smaller particle gels with re-crosslinkable bridges. Dynamic crosslinks such as hydrophobic associations, host-guest systems, electrostatic interactions and hydrogen bonding were used to generate self-healing hydrogels.[20] For example, copolymerization of hydrophobic stearyl methacrylate with long alkyl chain by micellar polymerization produced self-healing hydrogels based on dynamic hydrophobic associations.[21] In addition, cholic acid and $\beta$-cyclodextrin containing superamolecular hydrogels have self-healing ability from host-guest interactions.[22] However, most of self-healing hydrogels were incapable of re-forming bulk gels from the particle gels in brine as water was detrimental to the interactions from electrostatics and hydrophobicity and hydrogen bonding. However, polyion complex based hydrogels allowed for the formation of bulk gels in brine from polyion complex particles through reversible ionic bridges.[23-25] We proposed the particle gel based on ionic bonds can be re-crosslinked to generate bulk gel for sealing VSC containing reservoirs. Compared to tradition PPGs without recrosslinking ability to form the bulk gel through the particle gel, the re-crosslinkable PPGs could generate the bulk gel with comparable mechanical strength to traditional PPGs via polyion complex hydrogel.

Herein, we will show how re-crosslinkable hydrogels were synthesized by our unique approach due to the development of polyion complex hydrogels. The weak ionic bonds in-situ distributed within the gels provided self-healing, toughness, and adhesive properties to the synthetic cores. The ionic interactions within the hydrogels enabled us to prepare recrosslinkable mechanically robust hydrogels in brine from preformed particle gels. The mechanical strength of the re-formed hydrogels was quantified and compared with pristine bulk gels under reservoir conditions. The re-crosslinked particle gels showed excellent mechanical robustness and thermal stability in brine. The re-crosslinking ability in large fractured core to 
reduce water permeability was demonstrated by core flooding test in laboratory. This recrosslinkable particle gels offers the most promising materials up to date to increase conformance control in VSC containing reservoirs for fracture remediation.

\section{Experimental}

Materials. All chemicals and reagents were purchased from Sigma-Aldrich (St. Louis, MO) except as noted. Sodium Styrenesulfonate (Na-SS), 2-(Dimethylamino)ethyl methacrylate (DMAEMA) 2,2'-azobis(2-methylpropionamidine) dihydrochloride (V-50) were used as received. 2-acrylamido-2-methylpropanesulfonic acid (AMPS) was neutralized to generate corresponding sodium salt (Na-AMPS). [2-(Methacryloyloxy)ethyl] dimethylethylammonium chloride (Q-DMAEMA) monomer was prepared from DMAEMA monomer, which can be replaced by commercial [2-(Methacryloyloxy)ethyl] trimethylammonium chloride as purchased from Sigma-Aldrich. LiquiBlock ${ }^{\mathrm{TM}} 40 \mathrm{~K}$ superabsorbent gel, the crosslinked poly(acrylic acid) and poly(acrylamide) in potassium form, was obtained from Emerging Technologies inc. (Greensboro, NC)

Na-AMPS Salt Preparation. In laboratory scale, Na-AMPS in sodium salt form was prepared as follows. To a stirring solution of AMPS (103.5 g, $500 \mathrm{mmol})$ in ethanol (1000 mL) was added solid $\mathrm{NaOH}(20 \mathrm{~g}, 100 \mathrm{mmol})$ at room temperature. The reaction mixture was vigorously stirred for $1 \mathrm{~h}$ and Na-AMPS precipitated from ethanol. The product was filtered, washed with ethanol (100 mL x 3) and dried in vacuo at room temperature to give a white solid (105 g, Yield: $92 \%)$. 
Monomer Q-DMAEMA Synthesis. To a stirring solution of DMAEMA ( $47.1 \mathrm{~g}, 0.3 \mathrm{~mol})$ in acetonitrile $(150 \mathrm{~mL})$ was dropwise added bromoethane $(16 \mathrm{~g}, 0.6 \mathrm{mmol})$ at room temperature and stirred for $24 \mathrm{~h}$. The solution was concentrated under reduced pressure and the residual was triturated with hexane, filtered, dried in vacuo at room temperature to afford as a highly hygroscopic white solid Q-DMAEMA (24 g, Yield: $83 \%$ ) and used without further purification.

Re-crosslinkable Hydrogels Synthesis. All hydrogels were carried out in a similar fashion in glass vials. The hydrogels were prepared by polymerization of anionic monomers in the presence of cationic polymers. A typical hydrogel polymerization is as follows: a stirring solution of QDMAEMA (4.77 g, $18 \mathrm{mmol})$ and initiator V-50 in DI water (24 mL) was bubbled with argon for $30 \mathrm{~min}$ and kept in a preheated oil bath of $70{ }^{\circ} \mathrm{C}$ for $24 \mathrm{~h}$. To the as-prepared cationic polymer solution at room temperature were added anionic monomers of Na-AMPS (1.67 g, 8.1 mmol) and Na-SS $(2.27 \mathrm{~g}, 9.9 \mathrm{mmol})$ and kept in the oil bath of $70{ }^{\circ} \mathrm{C}$ for $24 \mathrm{~h}$ to generate the polyion complex hydrogels. The reaction conditions should be carefully tuned when performing the hydrogel synthesis due to their effects on mechanical and re-crosslinking properties. The resulting hydrogels were dried at $60{ }^{\circ} \mathrm{C}$ and processed to the particles in the size of $0.6 \mathrm{~mm}$ for further characterization and evaluations.

Rheological Studies. The rheological properties of hydrogels were measured using a Haake MARS III rheometer (Thermo Scientific Inc.). The disc-shaped samples in thickness of $\sim 2 \mathrm{~mm}$ and diameters of $15 \mathrm{~mm}$ were subject to rheological measurements at $25{ }^{\circ} \mathrm{C}$ using parallel-plate geometry (P35 Ti L) with a gap of $1 \mathrm{~mm}$. Strain sweep experiments were conducted to determine the linear strain regime. Oscillation time-dependent experiments were performed at a fixed 
frequency of $1 \mathrm{~Hz}$ and controlled strain of $1 \%$ to obtain shear elastic modulus of $\mathrm{G}^{\prime}$ and viscous modulus of $\mathrm{G}^{\prime \prime}$ as a function of time.

Morphology Characterization. Scanning electron microscopy images were collected on a HITACHI S-4700 FESEM microscope operating at $15.0 \mathrm{kV}$ to elucidate the microstructures of the gels. All images were captured with the samples after freeze-drying in non-hydrated state and sputter coated with $\mathrm{Au} / \mathrm{Pd}$ prior to imaging.

Core Physical Simulation Experiment. The plugging efficiency of the re-crosslinked gels were simulated and evaluated by core flooding experiment using large fractured sandstone, providing laboratory experimental basis for conformance control of field applications.

Table 1. Petrophysical properties of fractured core samples

\begin{tabular}{cccccccc} 
Core code & $\mathrm{L}(\mathrm{mm})$ & $\mathrm{D}(\mathrm{mm})$ & $\mathrm{W}_{\mathrm{f}}(\mathrm{mm})$ & $\mathrm{K}(\mathrm{mD})$ & $\Phi(\%)$ & $\mathrm{PV}\left(\mathrm{cm}^{3}\right)$ & $\mathrm{FV}\left(\mathrm{cm}^{3}\right)$ \\
\hline 1 & 110.5 & 50.4 & 1.91 & 44.73 & 22.0 & 47.5 & 6.63 \\
2 & 110.5 & 50.4 & 1.91 & 51.71 & 21.0 & 46.45 & 6.63 \\
\hline
\end{tabular}

Fractured Core Preparation. Natural sandstone cores were used to investigate plugging efficiency of the re-crosslinked bulk gel. The fractured cores were obtained by equal splitting approach.[26] The width, length and diameter of the fractured cores were directly measured due to the invalidity of empirical equation. The detailed information of the fractured core preparation was depicted in supporting information. The petrophysical parameters of the fractured cores were summarized in Table 1. 


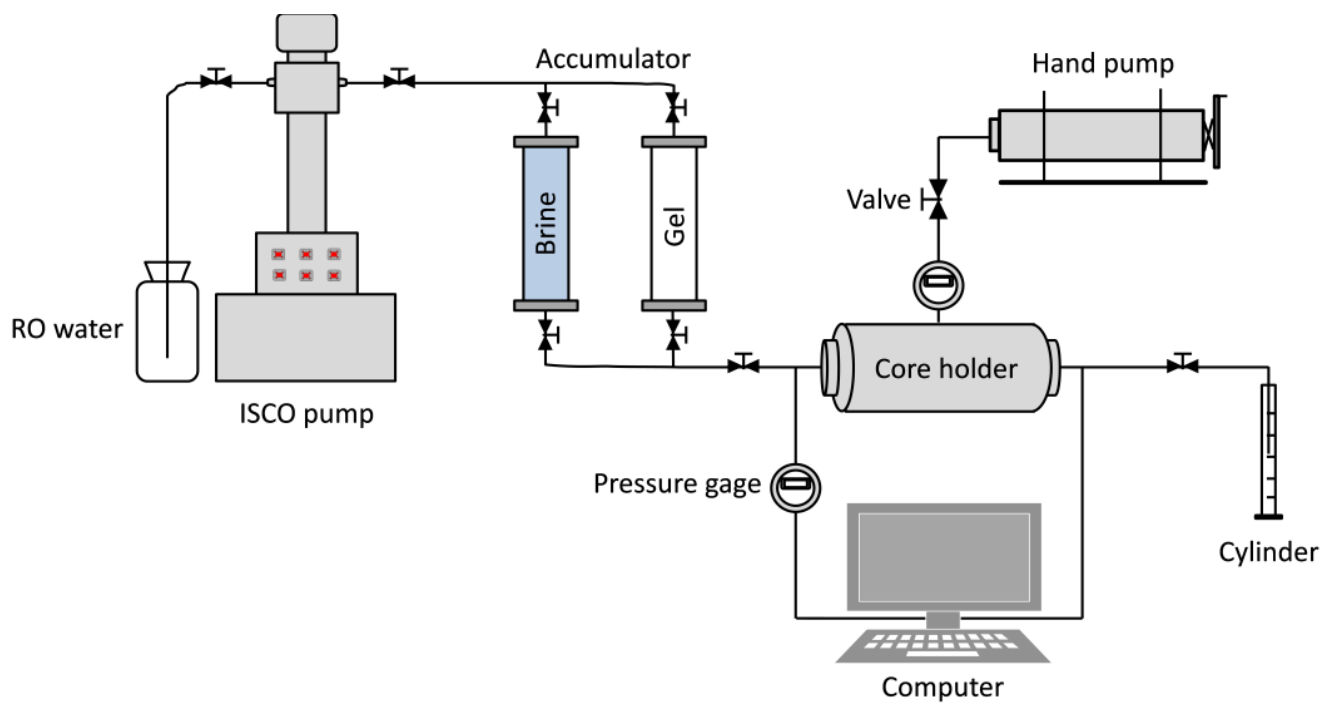

Scheme 1. Schematic diagram of single core equipment for dynamic physical stimulation.

Dynamic core flooding experiments were conducted following the equipment as shown in Scheme 1. Brine was injected to fractured core of sandstone at flow rate of $2 \mathrm{~mL} \mathrm{~min}^{-1}$, termed as first water flooding. To evaluate the plugging efficient of the re-crosslinkable particle gels, one fracture volume (FV) of the particle gels were placed to the fracture-aperture using modified procedure. The gel placement was in-situ regenerated to the bulk gel by a slurry of 6 wt $\% \mathrm{NaCl}$ brine at low-flowrate of $0.5 \mathrm{ml} \mathrm{min}^{-1}$ during which the particle gels were recrosslinked and in the fully swollen state as induced by brine. The injection flow rate of $6 \mathrm{wt} \%$ $\mathrm{NaCl}$ was raised up to $2 \mathrm{~mL} \min ^{-1}$ and the injection pressure was recorded to calculate the residual resistance factor (Frr) by following equation:

$$
F r r=\frac{P_{a}}{P_{b}}
$$

where $\mathrm{P}_{\mathrm{a}}$ and $\mathrm{P}_{\mathrm{b}}$ are the steady state pressure in psi during the first and second flooding, respectively. 


\section{Results and discussion}
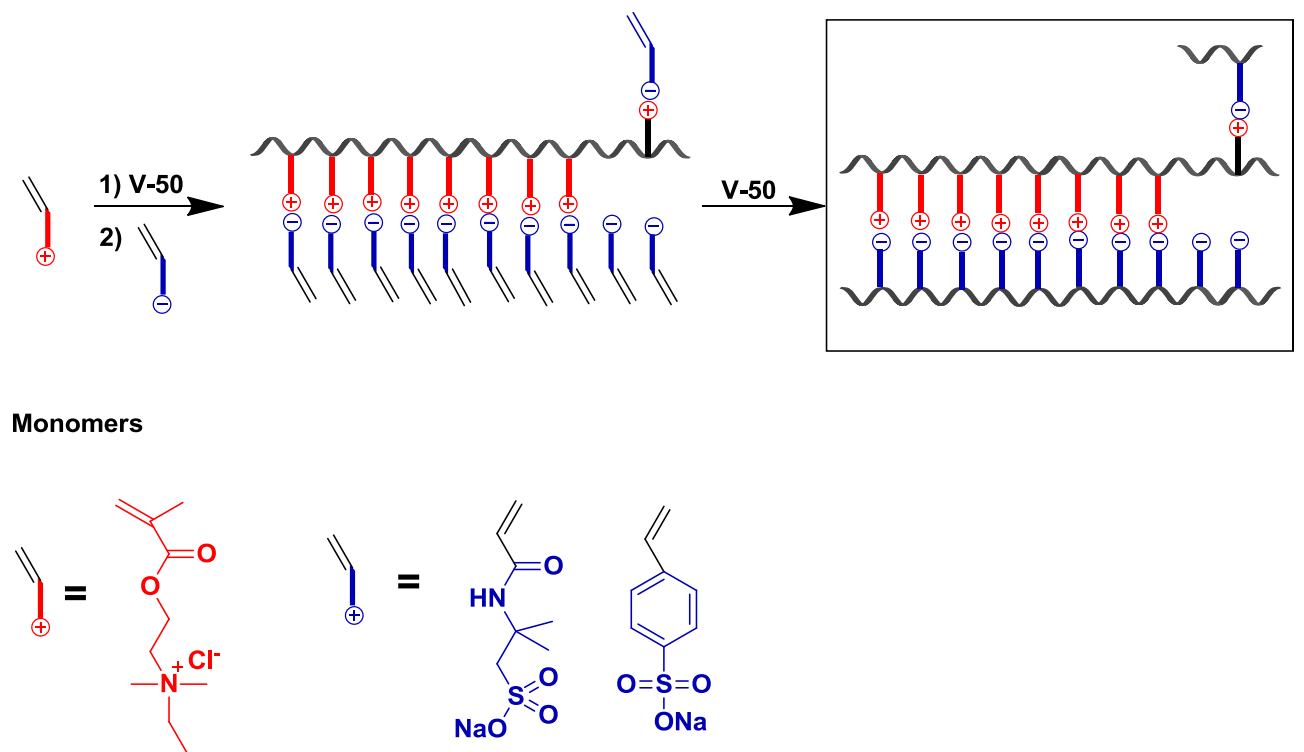

Scheme 2. General synthetic scheme of re-crosslinkable hydrogels from corresponding ionic synthons via sequential polymerization.

Preformed Particle Gels Preparation. The preformed particle gels having re-crosslinkable ability were prepared by the formation of polyion complexes via sequential polymerization of ionic monomers as borrowed from synthetic polymer chemistry. More explicitly, the hydrogels were fabricated from water soluble cationic PDMAEMA polymers in the presence of anionic Na-AMPS and Na-SS monomers under radical conditions as elaborated in this work as shown in Scheme 2. Tuning ionic strength of cationic moieties and anionic motifs generated brine responsive re-crosslinkable particle gels, which can be re-buildable above critical concentration of brine akin to reservoir conditions. Below the critical salt concentration of $0.5 \mathrm{wt} \%$, the recrosslinkable particles gels maintained intact under stirring conditions. This behavior would facilitate the gel injection and the treatment of in-depth reservoirs (vide infra). The strong bonding within the gels acted as permanent crosslinks while the weak interactions served as 
reversible sarcrificial ionic bonds, providing the re-crosslinkable features of the preformed particle gels.[23]

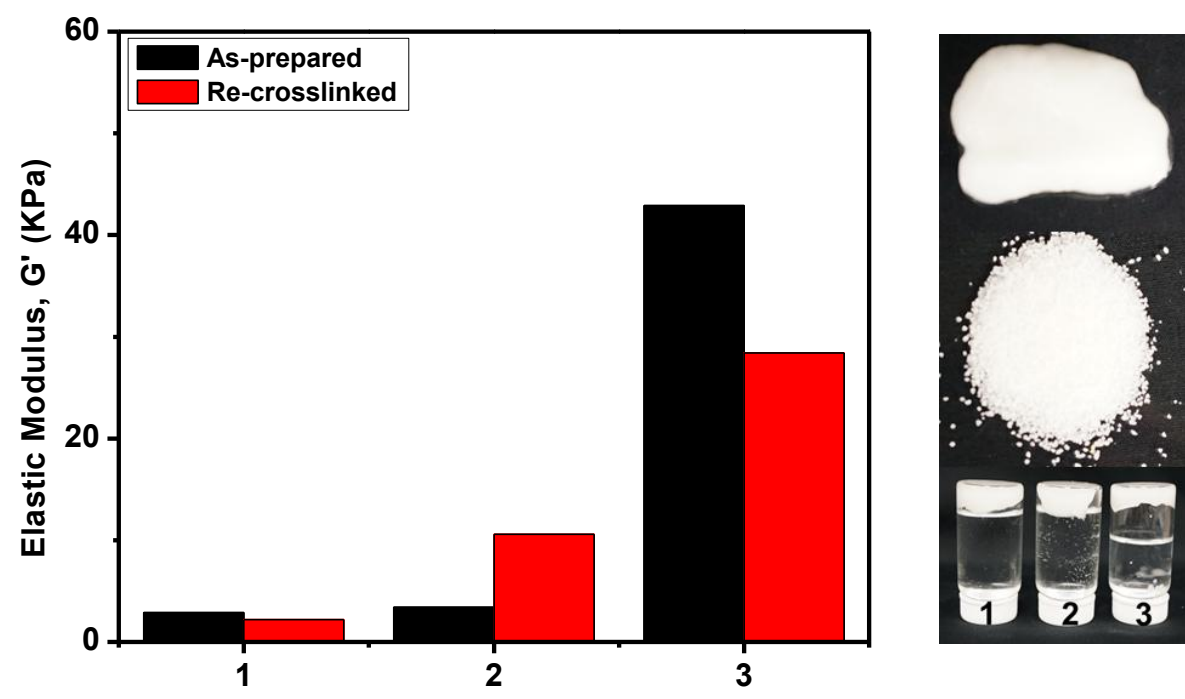

\section{Hydrogel}

Particle

Crosslinked

Figure 1. Elastic comparison of original bulk gels and re-formed bulk gels from particle gels under similar conditions. Sample 1, 2, 3 were Na-AMPS, Na-AMPS and Na-SS, Na-SS based gels, respectively. The particles of $1 \mathrm{~g}$ in the size of $0.6 \mathrm{~mm}$ in $1 \mathrm{wt} \% \mathrm{NaCl}$ of $\sim 20 \mathrm{~mL}$ were used to generate re-crosslinked bulk gels upon standing at room temperature for $12 \mathrm{~h}$.

Re-crosslinking Ability of the Particle Gels. The treatment of preformed particle gels to fractured reservoirs as a cost-effective approach has been demonstrated in mature water-flooded oil fields to correct permeability heterogeneity. However, the particle gels were unable to reform highly tough bulk gels due to the absence of interactions among the particles, rendering preformed particle gels ineffective plug high permeability zones/fractures.[17] Our unique hydrogels as demonstrated in this work are capable of re-forming bulk gels from particle gels as shown in Figure 1. We found the particle gels did not re-crosslink when the critical 
concentration of brine is less than $0.5 \mathrm{wt} \%$. Furthermore, the mechanical strength of the reformed particle gels was examined by rheometry. The as-prepared hydrogel is mechanically soft in physical appearance while the elasticity of the re-formed hydrogel is comparable to its original sample, even greater than the as-prepared hydrogel in the case of Na-AMPS and Na-SS based hydrogels. For example, the sample prepared from Na-AMPS or Na-SS monomers had lower mechanical strength after re-crosslinking compared to their counterparts. Interestingly, NaAMPS and Na-SS containing hydrogel showed higher elastic modulus of $10.6 \mathrm{KPa}$ than the asprepared sample with elasticity of $3.4 \mathrm{KPa}$. Probably the salt applied for the re-crosslinking induced the increase of the strong bonding formation within the gel for Na-AMPS and Na-SS sample. Based on the elastic moduli as indicated in Figure 1, the mechanical properties of the recrosslinkable hydrogels are one order magnitude larger than traditional poly(acrylamide) preformed particle gels, whose elasticity is in the range of $1000 \mathrm{~Pa}$

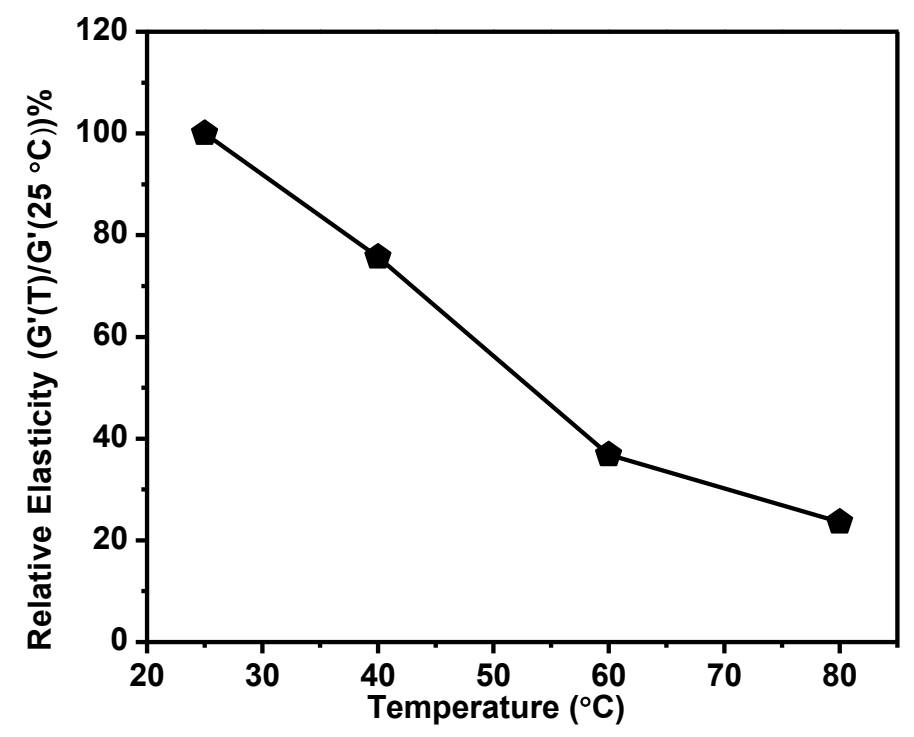

Figure 2. Temperature effect on gel integrity. The $0.6 \mathrm{~mm}$ particle gels were immersed in $1 \mathrm{wt} \%$ $\mathrm{NaCl}$ at the temperature of $25,40,60$ and $80{ }^{\circ} \mathrm{C}$ for $12 \mathrm{~h}$. 
Sample $2(1 \mathrm{~g})$ in the size of $0.6 \mathrm{~mm}$ in $1 \mathrm{wt} \% \mathrm{NaCl}(20 \mathrm{~mL})$ was cured at temperature of 25,40 , 60 and $80{ }^{\circ} \mathrm{C}$ for $12 \mathrm{~h}$ and the resulted re-crosslinked bulk gel was subject to rheological measurements at the corresponding aging temperature. As shown in Figure 2, the recrosslinkable PPG can form bulk gel at the temperature greater than $25{ }^{\circ} \mathrm{C}$ while the mechanical strength of bulk gel significantly reduced compared to the gel re-crosslinked at room temperature. This behavior would facilitate the injection of the particle gels under critical brine concentration. However, the particle gels are anticipated to re-form rubber-like bulk gels under reservoir conditions, which would improve plugging efficiency of the particle gels in field applications. . Given the re-crosslinkable merit and mechanical robustness, the re-crosslinked gel is a potentially excellent candidate to block high permeability fractures, thus increasing oil production by chemical enhanced oil recovery.

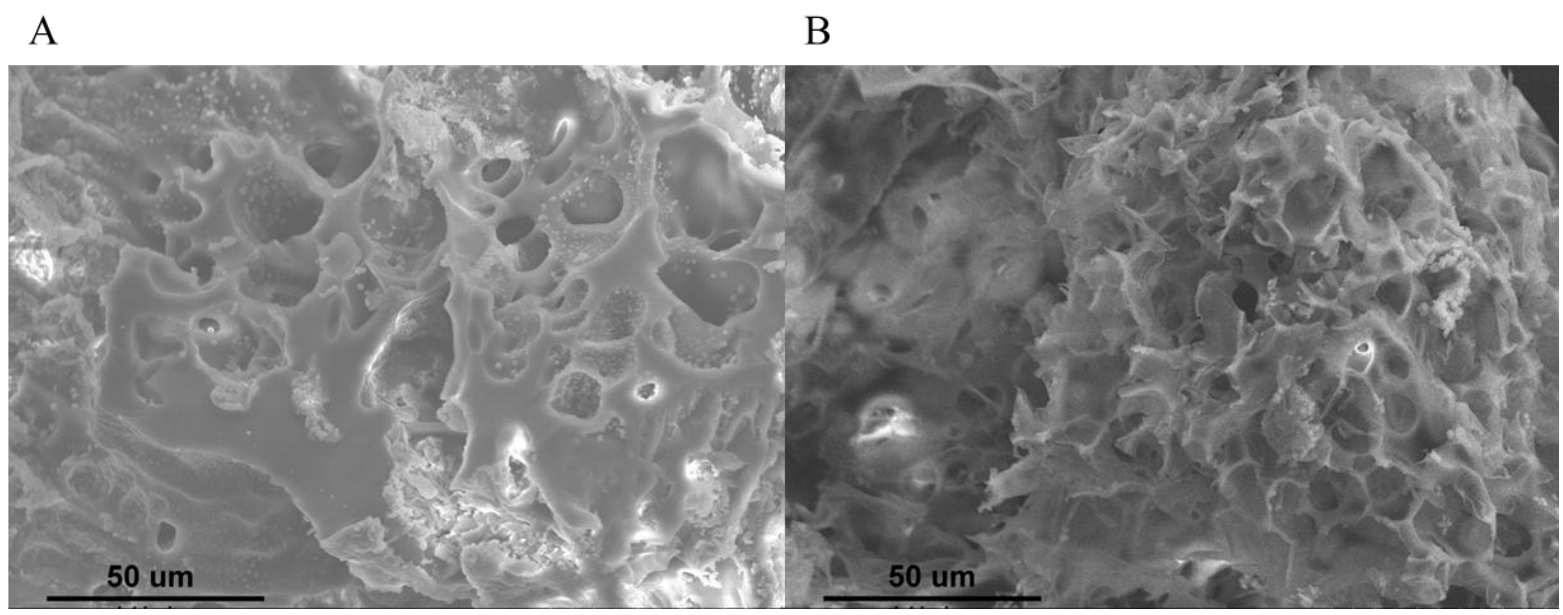

Figure 3. SEM micrographs of (A) pristine sample 2 prepared from cationic Q-DMAEMA and anionic monomers of Na-AMPS and Na-SS; (B) re-crosslinked gel from particle gel $\mathbf{2}$ in 1 wt \% brine at room temperature for $12 \mathrm{~h}$. 
Morphologies of Pristine and Re-crosslinked Gels. The pore sizes of the hydrogels in hydrated measurements are indicative of the gel strength. In our case, pore sizes imaged from SEM images of freeze-dried samples offered the estimation of the pore sizes in their fully swelling state. As observed in Figure 3, the re-crosslinked gel showed similar porous network to pristine sample. However, the pore size $(\sim 15 \mu \mathrm{m})$ of the re-crosslinked gel is slightly less than that of the pristine sample $(\sim 20 \mu \mathrm{m})$. The porosity of the hydrogels reflected the distance between crosslinking bridges. The morphology information partially explained the re-crosslinked sample demonstrated slightly higher elasticity of $10.6 \mathrm{KPa}$ than pristine gel of $3.4 \mathrm{KPa}$ as shown in Figure 1. This observation is well consistent with previous study in crosslinked poly(acrylamide) sample.[26] The pore size of hydrogel decreased with the concentration of the crosslinking points.

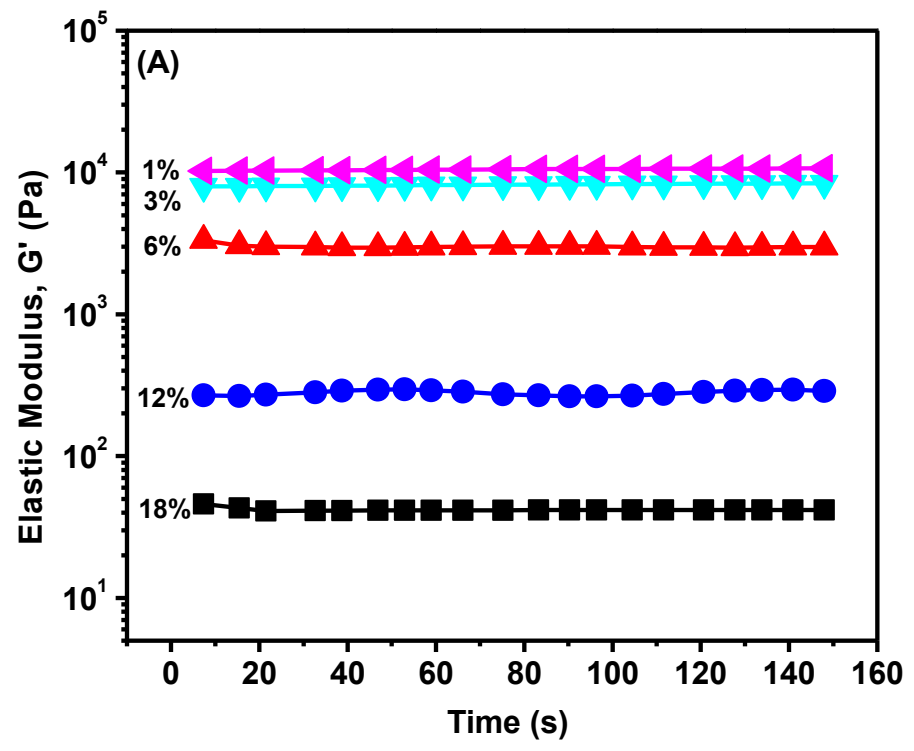

(B)

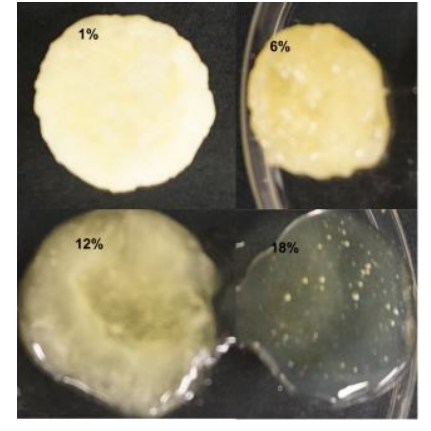

Figure 4. Time sweep profiles of shear elastic moduli of $\mathrm{G}^{\prime}$ at $25^{\circ} \mathrm{C}$ as a function of brine concentration, A: particle gel 2 treated with $1-18 \mathrm{wt} \% \mathrm{NaCl}$ for $12 \mathrm{~h}$; B: the images of the PPG in the size of $0.6 \mathrm{~mm}$ immersed in 1, 6, 12 and $18 \mathrm{wt} \% \mathrm{NaCl}$ at room temperature for $12 \mathrm{~h}$. 
Mechanical Strength of Re-crosslinked PPG Response to Brine. The mechanical strength of the re-formed bulk hydrogels are of great importance as plugging agents for conformance control, which would decrease reservoir heterogeneity and increase oil recovery. In our study, the shear elastic moduli of $\mathrm{G}^{\prime}$, a quantitative metric of gel strength, were measured as a function of time at a fixed frequency of $1 \mathrm{~Hz}$. The same amount of particle gels was cured in vials in brine for $12 \mathrm{~h}$ and subjected to rheological measurements at room temperature. As indicated in Figure $\mathbf{4 A}$, the mechanical strength of the re-formed bulk gel decreased as brine concentration increased. For example, the elastic modulus $\mathrm{G}^{\prime}, \sim 10 \mathrm{KPa}$ of the re-formed bulk gel in $1 \mathrm{wt} \% \mathrm{NaCl}$ was much greater than the sample in $6 \mathrm{wt} \% \mathrm{NaCl}$ with $\sim 3 \mathrm{KPa}$ elasticity. The reduction of the gel strength in re-formed bulk gel was due to the disruption of the weak interactions within the hydrogels induced by concentrated $\mathrm{NaCl}$ solution. In the polyion complexes based hydrogel, the multiple ionic bonds formed through inter- and intra-chain complexation played two roles as strong bonds and weak bonds. The strong bonds act as the permanent crosslinks while the weak bonds serve as reversible sacrificial bridges for hydrogel re-crosslinking (see Figure S1 in supporting information for more detailed explanations).[23,24] The significant decrease in mechanical robustness was observed as $\mathrm{NaCl}$ concentration reached up to $18 \mathrm{wt} \%$, whose elasticity was 0.04 KPa decreased by a factor more than 1000. Apparently as shown in Figure 4A, the bulk gel strength relied on brine concentration. These observations were in consistent with the physical appearance of the re-formed bulk gels. The bulk gel maintained mechanical integrity in $1 \mathrm{wt} \%$ brine while brine softening effect on re-formed bulk gels was observed with increased brine concentration in Figure 4B as the gels became transparent and flexible. As implied in Figure S2, the crosslinked gel maintained mechanical integrity over the extended timescale of $1800 \mathrm{~s}$ in our rheological measurements. The sample showed constant elasticity of $\sim 10 \mathrm{KPa}$ in the range of 
$1800 \mathrm{~s}$, which is similar to the gel strength at the shorter time period of $150 \mathrm{~s}$. Compared to gellike conglomeration prepared from salt tolerant modified starch and hydrolyzed poly(acrylamide) with viscosity of less than $1000 \mathrm{mP} . \mathrm{S}$ having in-situ gel features, the re-crosslinked gels showed superior mechanical behavior with elasticity of $10 \mathrm{KPa}$.[27] In addition, the re-crosslinking ability and gel strength of the re-crosslinkable gels were independent of the gel concentration in brine, which was in stark contrast to the mechanical properties of in-situ gel as determined by gelant concentration. For example, the re-crosslinked bulk gel showed similar elasticity of $\sim 10 \pm$ $1.0 \mathrm{kPa}$ under particle gel concentration at 2.5, 5 and $10 \mathrm{wt} \%$ in $1 \mathrm{wt} \% \mathrm{NaCl}$.

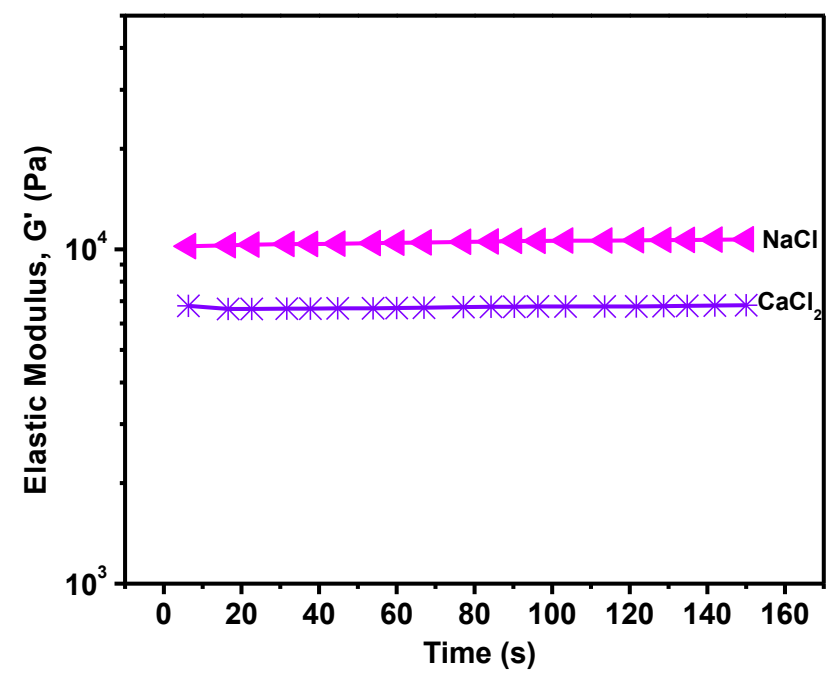

Figure 5. Comparison of mechanical strength of the particle gel re-formed in $\mathrm{NaCl}$ and $\mathrm{CaCl}_{2}$ solution. The bulk gel was formed by addition of sample 2 of $1 \mathrm{~g}$ in $1 \mathrm{wt} \% \mathrm{NaCl}$ and $\mathrm{CaCl}_{2}$ of 20 $\mathrm{mL}$ upon standing at room temperature for $12 \mathrm{~h}$.

High salinity of Ordovician cave-fractured carbonate reservoir such as Tahe oilfield had an adverse effect on PPG, which dramatically reduce swelling ratio and stability of the gels.[28,29] Salt tolerance of the re-formed particle gel was examined under different salt solutions. We found the particle gel had the re-crosslinkable ability in the presence of divalent 
ions. As shown in Figure 5, the re-crosslinked bulk gel in $1 \mathrm{wt} \% \mathrm{CaCl}_{2}$ had comparable elastic modulus of $7 \mathrm{KPa}$ to the sample with $10 \mathrm{KPa}$ in $1 \mathrm{wt} \% \mathrm{NaCl}$. The situation was radically different from $\mathrm{Cr}^{3+}$ crosslinked conventional partially hydrolyzed poly(acrylamide) in $1 \mathrm{wt} \%$ $\mathrm{NaCl}$ with elastic modulus of $9.0 \mathrm{~Pa}$, which had higher elasticity of $14.0 \mathrm{~Pa}$ in $1 \mathrm{wt} \% \mathrm{CaCl}_{2}$ solution due to the contraction of the gels from screening effect.

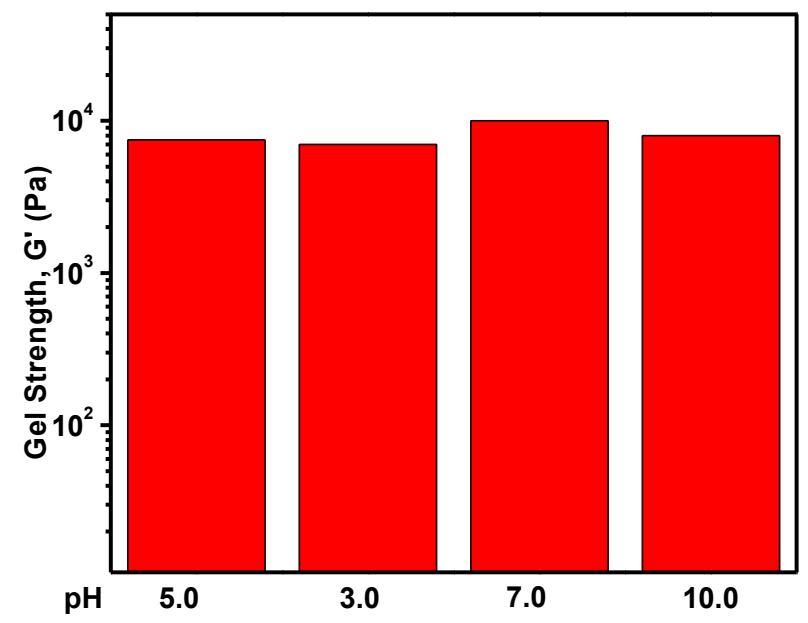

Figure 6. Re-crosslinked gel strength as a function of $\mathrm{pH}$ at room temperature in $1 \mathrm{wt} \% \mathrm{NaCl}$. The bulk gel was formed by addition of $0.6 \mathrm{~mm}$ PPG of $1 \mathrm{~g}$ in $1 \mathrm{wt} \% \mathrm{NaCl}$ upon standing at room temperature for $12 \mathrm{~h}$.

The re-crosslinking ability and mechanical integrity of the particle gels were examined under reservoir conditions with different pHs. Commercial LiquiBlock ${ }^{\mathrm{TM}} 40 \mathrm{~K}$ gel had lower swelling capacity of $15 \mathrm{~g} / \mathrm{g}$ at $\mathrm{pH}$ of 3.0 compared to its swelling ratio of $250 \mathrm{~g} / \mathrm{g}$ at $\mathrm{pH}$ of 7.0 in water. In this work, the re-crosslinking ability and gel strength were visually observed and evaluated by rheometry. We found our re-crosslinkable particle gels can form mechanically robust bulk gel in acidic and alkaline environments ranging from 3.0 to 10.0. Furthermore, the 
re-formed particle gels maintained its mechanical integrity as demonstrated by their similar elastic moduli in Figure 6, albeit the re-formed gels showed slightly lower elasticity in acidic or alkaline conditions. These data implied that the mechanical strength of the gels was insensitive to $\mathrm{pH}$ of reservoir environments.
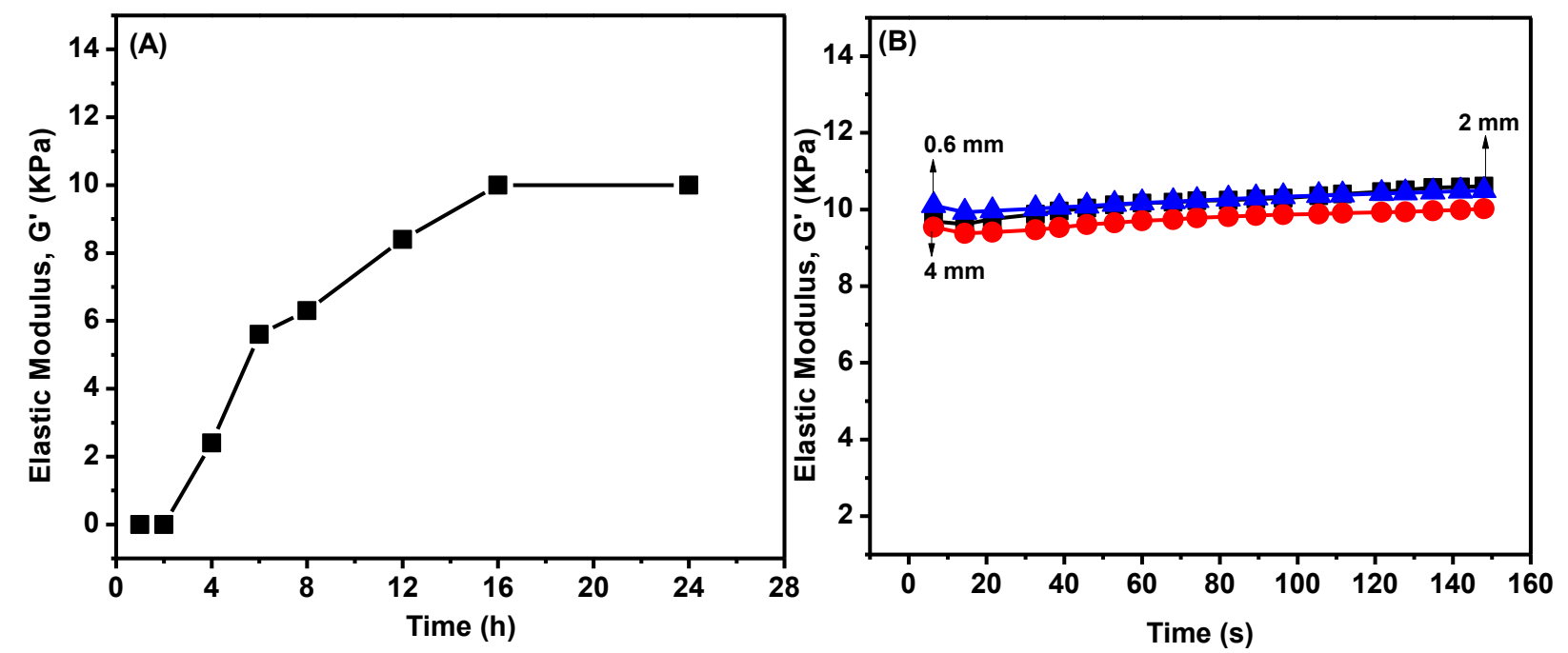

Figure 7. (A) re-crosslinking kinetics of the particle gel of $0.6 \mathrm{~mm}$ and (B) mechanical strength of the particle gels in the size of $0.6,2.0$ and $4.0 \mathrm{~mm}$ from top to bottom in $1 \mathrm{wt} \% \mathrm{NaCl}$ at room temperature for $12 \mathrm{~h}$.

Re-crosslinking kinetics of the particle gel were key parameters for injectivity and plugging efficiency after placement of particle gels in conduits. Early re-crosslinking of the particle gel during pumping resulted in the difficulty of injection and short distance transportation to the placement. In our study, the re-crosslinking kinetics was monitored as a function of time by rheological measurements. The kinetics of re-crosslinking process also depends upon the particle size and the salinity of the reservoirs. As demonstrated in Figure 7A, the particle gels of $0.6 \mathrm{~mm}$ in dry white granular form immersed in $1 \mathrm{wt} \% \mathrm{NaCl}$ vials regenerated bulk gels after $2 \mathrm{~h}$. The gels in the size of $4 \mathrm{~mm}$ formed re-crosslinked bulk gels for 8 
$\mathrm{h}$ under similar environments. The elasticity of the re-crosslinked gels increased over time and maintained constant after $16 \mathrm{~h}$, which indicated the particle gels were in their maximal crosslinking state. However, we found the re-crosslinkable particle gel maintained their initial shape under vigorous stirring, indicative of the absence of crosslinking links among the particles in dynamic conditions due to the absence of close contact. The mechanical strength of the recrosslinked gel was not controlled by the size of the re-crosslinkable particle gels as they had similar elastic moduli as shown in Figure 7B. Thus, our approach has high fidelity in recrosslinking kinetic control by tuning the particle size and facilitates the injection upon pumping.
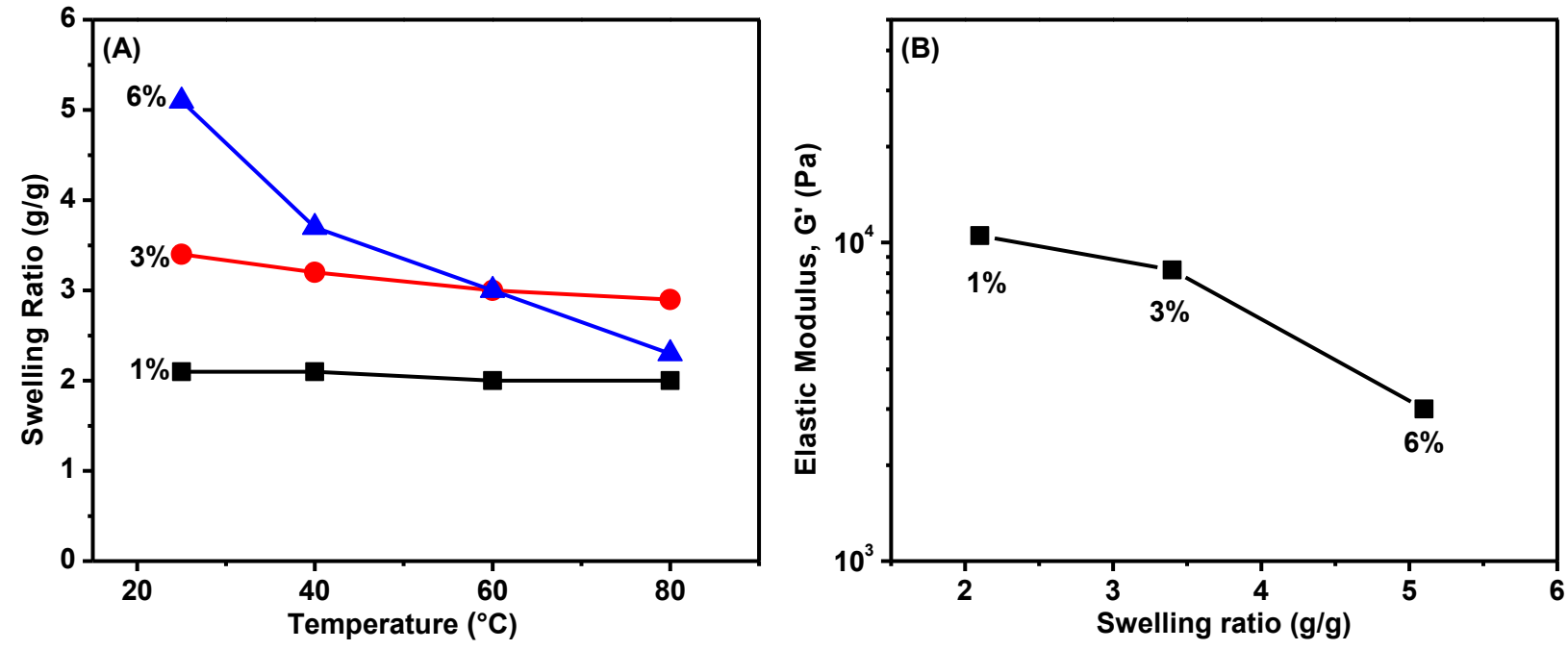

Figure 8. (A) Swelling ratio of the re-crosslinked gel aged for 6 months as a function of temperature in aqueous $\mathrm{NaCl}$ solution, (B) mechanical strength of the bulk gels at room temperature in brine.

Swelling Behaviors and Thermal Stability. Long-term thermal stability under reservoir conditions is the critical parameter of the particle gel as the plugging agent for chemical enhanced oil recovery. In this study, swelling ratio of the re-crosslinked bulk gel in brine was employed to monitor the thermal stability of the re-crosslinked gel. As indicated in Figure 8A, 
the swelling ratio of the re-crosslinked bulk gel increased with the increase of the salt concentration at room temperature, probably due to entanglement reduction of the ionic polymer chains disrupted by salt (see Figure $\mathbf{S 1}$ in supporting information). At $\mathrm{NaCl}$ concentration lower than $3 \mathrm{wt} \%$ the re-crosslinked gel in brine maintained its swelling ratio at room temperature as the temperature increased to $80{ }^{\circ} \mathrm{C}$, showing excellent salt resistance. However, the swelling ratio of the re-crosslinked hydrogels markedly decreased at salt concentration of $6 \mathrm{wt} \%$ as the temperature was raised to $80{ }^{\circ} \mathrm{C}$. The swelling behaviors of the re-crosslinked particle gel are radically different to conventional PPG, whose swelling ratio increases with temperature and significantly decrease with salt concentration. Furthermore, as shown in Figure S3, the recrosslinked gel showed similar swelling ratios in $\mathrm{NaCl}$ and $\mathrm{CaCl}_{2}$ solutions with concentrations ranging from 1 to $6 \mathrm{wt} \%$. Compared to conventional PPG, the ionic crosslinked PPG showed excellent resistance to divalent ions of $\mathrm{CaCl}_{2}$. For example, the swelling ratio of LiquiBlock ${ }^{\mathrm{TM}}$ $40 \mathrm{~K}$ in $1 \mathrm{wt} \% \mathrm{NaCl}$ is approximately $35 \mathrm{~g} / \mathrm{g}$ while in $1 \mathrm{wt} \% \mathrm{CaCl}_{2}$ the swelling ratio decreases to $17.5 \mathrm{~g} / \mathrm{g}$. Further work on improving swelling ratio in low concentration of brine is under our scrutiny.

In consistent with short term evaluation for $12 \mathrm{~h}$, the swelling ratio of the bulk gel was almost constant over 6 months when the brine concentration was less than 3 wt $\%$. Apparently, our re-crosslinkable particle gel falls into the scenario of low temperature, high salinity oilfields in Alaska given its moderate swelling ratio greater than 5.0 and long-term thermal stability for 6 months. Furthermore, as indicated in Figure 8B, the elasticity of the re-crosslinked bulk gel decreased as the swelling ratio increased due to the reduction of ionic crosslinks. However, the re-crosslinked hydrogel showed three times mechanical strength of $\sim 3000 \mathrm{~Pa}$ compared to commercial LiquiBlock $^{\mathrm{TM}} 40 \mathrm{~K}$ gel with elasticity of $\sim 1000 \mathrm{~Pa}$. The concentration of further 
work would be on the improvement of salt resistance by introducing covalent crosslinks during particle gel synthesis.
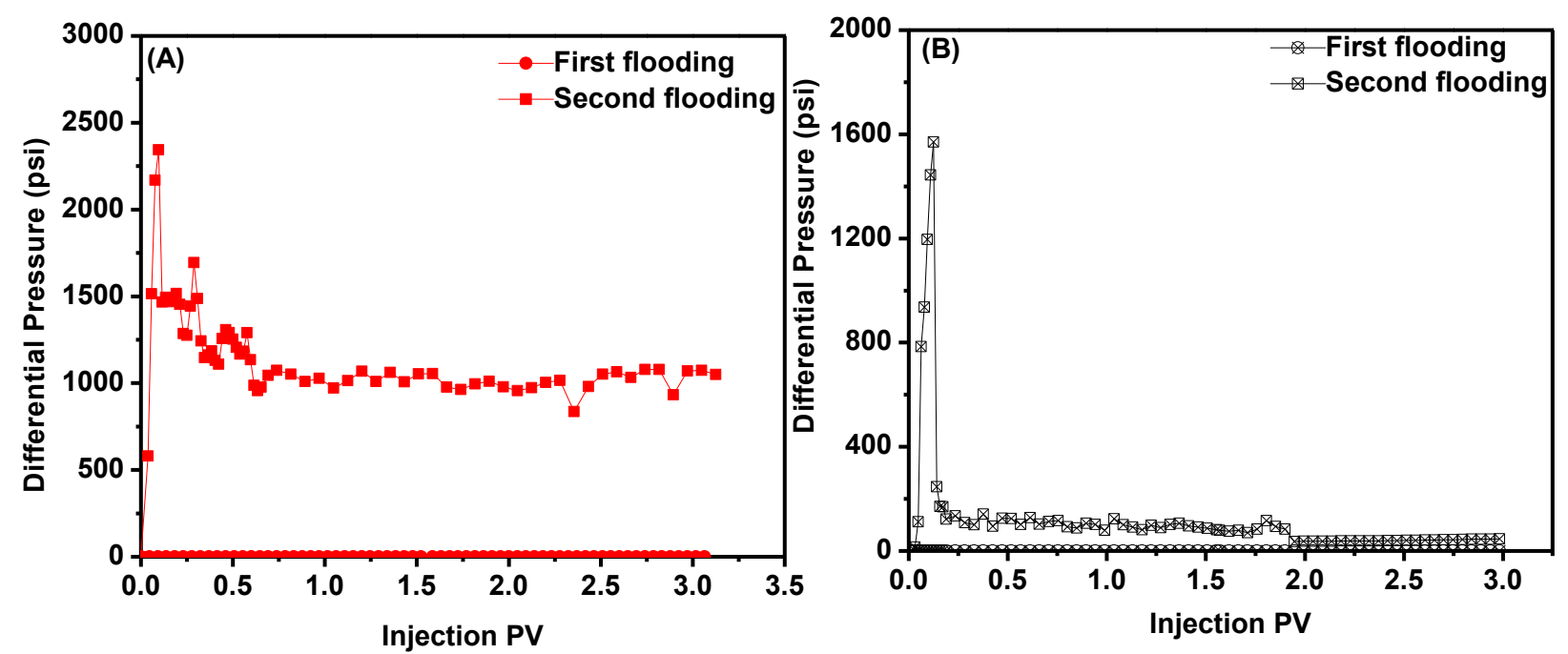

Figure 9. Differential pressure as a function of injection fracture volume, (A) dynamic physical simulation of the re-crosslinked bulk gel, (B) core flooding experiment of Liquidblock ${ }^{\circledR} 40 \mathrm{~K}$ gel.

Synthetic fractured core model provides a viable approach to predict the performance of the preformed particle gel under reservoirs in a timely fashion. Generally, field applications of gel treatment were constrained by funding stringency and time availability. In this study, residual resistance factor, a critical parameter of particle gel plugging behavior to water, was employed to evaluate the plugging efficiency of the re-crosslinked bulk gel. The significant differential pressure increase demonstrated the gel efficiently block the fractures. As shown in Figure 9, the re-crosslinked bulk gel had breakthrough pressure of 2344 psi, which was greater than LiquiBlock $^{\mathrm{TM}} 40 \mathrm{~K}$ gel with 1570 psi. The initial pressure increase could be ascribed to the absence of brine transport pathway within the gels while the differential pressure suddenly decreased due to the formation of wormholes or new permeable paths. This phenomenon is similar to the work as reported by $\mathrm{Pu}$, et al.[30] In addition, the re-crosslinked bulk gel had one 
order of magnitude higher residual resistance factor of 4510.5 compared to commercial $40 \mathrm{~K}$ gel having 193.5 resistance factor. Furthermore, the higher residual resistance factor was also demonstrated by higher differential pressure under steady flow state. During dynamic physical experiments we observed that commercial $40 \mathrm{~K}$ gel was extruded from the fractured core due to its weak mechanical integrity as observed in previous research [31] while the re-crosslinked bulk gel showed good resistance to brine under the pressure of 1013.5 psi after $0.5 \mathrm{PV}$. The extrusion of the commercial LiquiBlock ${ }^{\mathrm{TM}} 40 \mathrm{~K}$ gel was further validated by the differential pressure decrease on a continuous basis after approximately 2 injection PV, during which the differential pressure dropped from $108 \mathrm{psi}$ at $\sim 0.3 \mathrm{PV}$ via $80 \mathrm{psi}$ at $\sim 1.1 \mathrm{PV}$ to constant pressure of $40 \mathrm{psi}$ after 2 PV. The core flooding experiment indicated the fractured core can be blocked by the recrosslinked gel, which showed excellent plugging performance to brine flow.

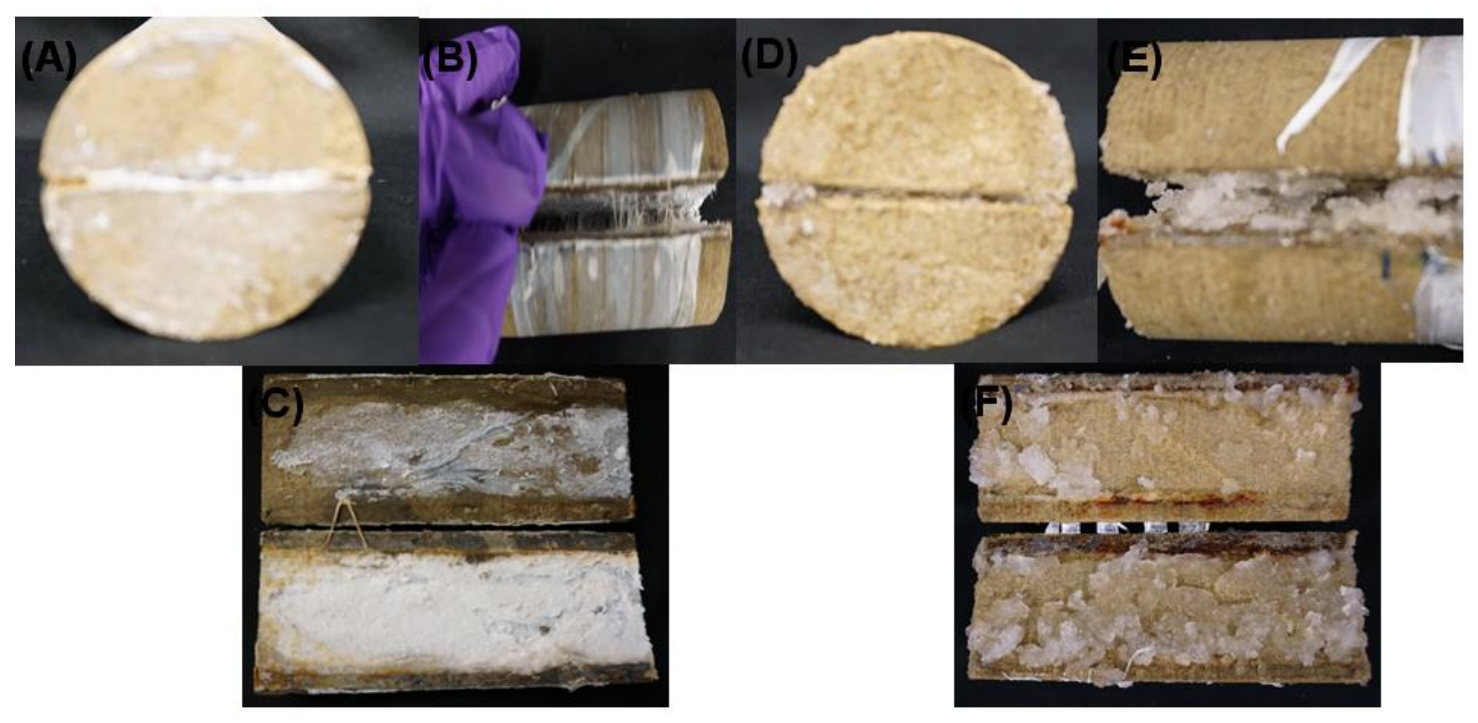

Figure 10. Images of the gels after dynamic physical simulation in different angles. (A), (B) and (C) are the pictures of the re-crosslinked gel; (D), (E) and (F) are the profiles of $40 \mathrm{~K}$ gel. 
The morphology of the gels after core flooding experiment was examined to correlate the plugging efficiency with macroscopic structures. As shown in Figure 10A the cross-section of the synthetic fracture was completely sealed by the re-crosslinked bulk gel while $40 \mathrm{~K}$ gel partially plugged the fracture in physical appearance (Figure 10D). In addition, the recrosslinked bulk gel had strong adhesive interaction with the fracture surface after core flooding experiment, which was stretchable (Figure 10B). However, there is no apparent adhesion between $40 \mathrm{~K}$ gel and fractured core as observed in Figure 10E. As observed in Figure S4A, the re-crosslinkable particle gel suspension solution at the concentration of $1 \mathrm{wt} \%$ in $0.5 \mathrm{wt} \%$ brine had water-like low viscous behavior. The suspension solution maintained its initial uncrosslinked state under stirring for $12 \mathrm{~h}$ (Figure S4B). As demonstrated in the laboratory study, under dynamic stirring conditions for $12 \mathrm{~h}$ the re-crosslinkable gel would not re-form bulk gel probably due to the absence of close contact among individual particle gel as shown in Figure S4B. Furthermore, the re-crosslinked bulk gel maintained mechanical integrity after dynamic physical simulation in Figure 10C. The integrity ensured the re-crosslinked bulk gel had the excellent washout resistivity compared to $40 \mathrm{~K}$ as demonstrated in core flooding experiment. The wormholes of $40 \mathrm{~K}$ gel distributed in an arbitrary fashion were observed within the gel in Figure 10F. The wormholes further elucidated the lower differential pressure during core flooding experiment.

\section{Conclusions}

In conclusion, the preformed particle gel with re-crosslinkable ability to re-form bulk gel for chemical enhanced oil recovery of large fractured reservoir has been described. The formation of bulk gel was achieved by reversible ionic crosslinks of the preformed particle gels 
as induced by brine under reservoir conditions. The re-crosslinked bulk gel with ionic features showed mechanically robust behaviors. The re-crosslinking ability of the preformed particle gel under different salinity can be controlled by tuning the ionic interactions of monomer pairs. In addition, the long-term thermal stability of the re-crosslinked bulk gel has been demonstrated. Furthermore, the re-crosslinked bulk gels can significantly reduce permeability of large fractures as demonstrated by core flooding experiments. The re-crosslinkable bulk gel with robust mechanical properties offered the candidate to block large fractured reservoirs for chemical enhanced oil recovery.

\section{Associated Content}

Supporting Information. Detailed preparation of the fractured core is provided.

\section{Author Information}

Corresponding author. *Email: wangliz@mst.edu, baib@mst.edu

\section{Acknowledgements}

The authors would like to express their grateful acknowledge to the financial support from DOE under the contract of DE-FE0024558

\section{References}

1. R.C.Jr. Fielding, G.H. Gibbons, F.P. Legrand, In-depth drive fluid diversion using an evolution of colloidal dispersion gels and new bulk gels: an operational case history of north rainbow ranch unit. presented at the SPE/DOE improved oil recovery symposium, Tulsa, Oklahoma, April 17-20, 1994; Paper SPE 27773.

2. S. McCool, X.P. Li, G.P. Willhite, Flow of a Polyacrylamide/Chromium Acetate System in a Long Conduit. SPE J. 14(1) 2009 54-66. 
3. L. Montanari, R. Scotti, T.P. Lockhart, Kinetics and Mechanism of the Reaction of Hydrated Chromium (II1) with Partially Hydrolyzed Polyacrylamide. Macromolecules 271994 3341-3348.

4. R.D. Sydansk, G.P. Southwell, More than 12 years' experience with a successful conformance-control polymer-gel technology. SPE Prod. \& Oper. 15(4) 2000 270-278.

5. Y. Liu, C. Dai, K. Wang, M. Zhao, M. Gao, Z. Yang, J. Fang, Y. Wu, Investigation on preparation and profile control mechanisms of the dispersed particle gels (DPG) formed from phenol-formaldehyde cross-linked polymer gel. Ind. Eng. Chem. Res. 552016 6284-6292.

6. C. Dai, W. Chen, Q. You, H. Wang, Z. Yang, L. He, B. Jiao, Y. Wu, A novel strengthened dispersed particle gel for enhanced oil recovery application. J. Ind. Eng. Chem. 412016 175182.

7. X. Chen, Q. Feng, W. Liu, K. Sepehrnoori. Modeling preformed particle gel surfactant combined flooding for enhanced oil recovery after polymer flooding. Fuel 1942017 42-49.

8. J.P. Coste, Y. Liu, B.J. Bai, Y. Li, P. Shen, Z. Wang, G. Zhu, In-depth fluid diversion by pregelled particles. Laboratory study and pilot testing. Presented at the SPE/DOE improved oil recovery symposium, Tulsa, Oklahoma, April 3-5, 2000; Paper SPE 59362.

9. D.D. Smith, M.J. Giraud, K.M. McBee, J.A. Taitano, M.S. Winfield, J.T. Portwood, D.M. Everett, The successful evolution of Anton Irish conformance efforts. Presented at the SPE annual technical conference \& exhibition, San Antonio, Texas, U.S.A., September 24-27, 2006; Paper SPE 103044.

10. T.L. Hughes, F. Friedmann, D. Johnson, G.P. Hild, A. Wilson, S.N. Davies, Large-volume foam-gel treatments to improve conformance of the Rangely $\mathrm{CO}_{2}$ flood. SPE Reservoir Eval. \& Eng. 2(1) 1999 14-24.

11. Ǿ. Eide, M.A. Ferno, Z. Alcorn, A. Graue, Visualization of carbon dioxide enhanced oil recovery by diffusion in fractured chalk. SPE J. 21(3) 2016 112-120.

12. R.S. Seright, Use of preformed gels for conformance control for fractured system. SPE Prod. \& Oper. 12(1) 1997 59-65.

13. R.H. Lane, R.S. Seright, Gel water shutoff in fractured or faulted horizontal wells. Presented at the SPE/CIM international conference on horizontal well technology, Calgary, Alberta, Canada, November 6-8, 2000; paper SPE 65527.

14. A. Al-lbadi, F. Civan, Experimental investigation and correlation of treatment in weak and high-permeability formations by use of gel particles. SPE Reservoir Eval. \& Eng. 28(4) 2013 387-401. 
15. M. Kiani, H. Kazemi, E. Ozkan, Y.S. Wu, Pilot testing issues of chemical EOR in large fractured carbonate reservoirs. Presented at the SPE annual technical conference \& exhibition, Denver, Colorado, U.S.A., October 30 - November 2, 2011; paper SPE 146840.

16. X.N. Xie, W.W. Weiss, Z.X. Tong, N.R. Morrow, Improved oil recovery from carbonate reservoirs by chemical stimulation. SPE J. 10(3) 2005 276-285.

17. B.J. Bai, L.X. Li, Y.Z. Liu, L. He, Z.G. Wang, C.M. You, Preformed particle gel for conformance control: factors affecting its properties and applications. SPE Reservoir Eval. \& Eng. 10(4) 2007 415-422.

18. B.J. Bai, Y.Z. Liu, J.P. Coste, L.X. Li, Preformed particle gel for conformance control: transport mechanism through porous media. SPE Reservoir Eval. \& Eng. 10(2) 2007 176-184.

19. H. Zhang, B.J. Bai, Preformed Particle Gel Transport through open fractures and its effect on water flow. SPE J. 152010 389-400.

20. I. Jeon, J.X. Cui, W.R. Illeperuma, J. Aizenberg, J. Vlassak, Extremely stretchable and fast self-healing hydrogels. Adv. Mater. 282016 4678-4683.

21. V. Can, Z. Kochovski, V. Reiter, N. Severin, M. Siebenburger, B. Kent, J. Just, J.P, Rabe, M. Ballauff, O. Okay, Nanostructural evolution and self-healing mechanism of micellar hydrogels. Macromolecules $4920162281-2287$.

22. L. Huang, N. Yi, Y. Wu, Y. Zhang, Q. Zhang, Y. Huang, Y. Ma, Y. Chen, Multichannel and repeatable self-healing of mechanical enhanced graphene-thermoplastic polyurethane composites. Adv. Mater. 252013 2224-2228.

23. F. Luo, T.L. Sun, T. Nakajima, T. Kurokawa, Y. Zhao, K. Sato, A.B. Ihsan, X. Li, H. Guo, J.P. Gong, Oppositely charged polyelectrolytes form tough, self-healing, and rebuildable hydrogels. Adv. Mater. 272015 2722-2727.

24. T.L. Sun, T. Kurokawa, S. Kuroda, A.B. Ihsan, T. Akasaki, K. Sato, M.A. Haque, T. Nakajima, J.P. Gong, Physical hydrogels composed of polyampholytes demonstrate High toughness and viscoelasticity. Nat. Mater. 122013 932-937.

25. F. Luo, T.L. Sun, T. Nakajima, T. Kurokawa, A.B. Ihsan, X. Li, H. Guo, J.P. Gong, Free reprocessability of tough and self-healing hydrogels based on polyion complex. ACS Macro Lett. 42015 961-964.

26. J.H. Wen, L.G. Vincent, A. Fuhrmann, Y.S. Choi, K.C. Hribar, H.T. Weiner, S. Shen, A.J. Engler, Interplay of matrix stiffness and protein tethering in stem cell differentiation. Nat. Mater. 132014 979-987. 
27. C.L. Dai, S. Yang, X.P. Wu, D.X. Peng, K. Wang, Y.N. Wu, Investigation on polymer reutilization mechanism of salt-tolerant modified starch on offshore oilfield Energy Fuels 30 $20165585-5592$.

28. C.D. Yuan, W.F. Pu, F.Y. Jin, Y.C. Zhang, H. Jia, T.H. Zhao, Performance of oil-Based cement slurry as a selective water-plugging agent in high-temperature and high-salinity cavefractured carbonate reservoirs. Ind. Eng. Chem. Res. 532014 6137-6149.

29. Y.R. Bai, F.L. Wei, C.M. Xiong, J.J. Li, R.Y. Jiang, H.B. Xu, Y. Shu, effects of fracture and matrix on propagation behavior and water shut-off performance of a polymer gel. Energy Fuels 292015 5534-5543.

30. J.Z. Zhao, H. Jia, W.F. Pu, R. Liao, Influences of Fracture Aperture on the water-shutoff performance of polyethyleneimine cross-linking partially hydrolyzed polyacrylamide gels in hydraulic fractured reservoirs. Energy Fuels 252011 2616-2624.

31. W.F. Pu, C.L. Wen, R. Liu, F.Y. Lin, C.Y. Wang, Z.C. Liao, Evaluation of a novel profile control agent for enhancing an oil-recovery application J. Appl. Poly. Sci. 1332016 4375643766. 


\title{
Mechanically Robust Re-crosslinkable Polymeric Hydrogels for Water Management of Void Space Conduits Containing Reservoirs
}

\author{
Lizhu Wang,* Yifu Long, Haifeng Ding, Jiaming Geng, Baojun Bai*
}

Department of Geosciences and Geological and Petroleum Engineering, Missouri University of Science and Technology, Rolla, MO 65409, USA

*To whom correspondence should be addressed. E-mail:wangliz@mst.edu, baib@mst.edu

Table of Contents Graph

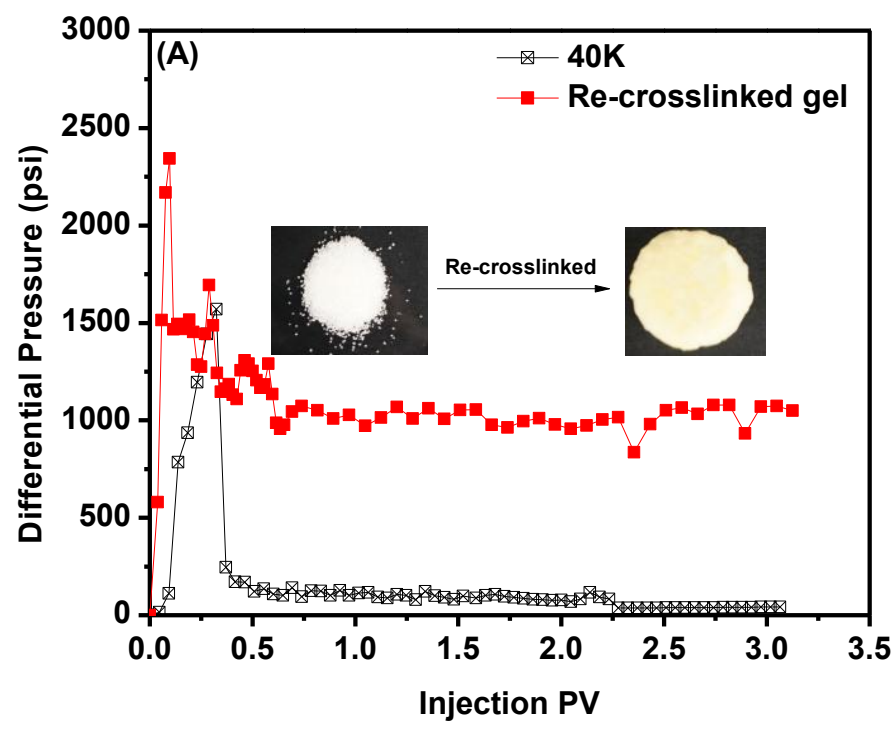

